\title{
Forecasting for Day-ahead Offshore Maintenance Scheduling under Uncertainty
}

\author{
J. Browell, I. Dinwoodie \& D. McMillan \\ Department of Electronic and Electrical Engineering \\ University of Strathclyde, Glasgow, UK
}

\begin{abstract}
Offshore wind farm maintenance operations are complex and dangerous, and as such are subject to strict safety constraints. In addition, crew and vessels must be scheduled in advance for both planned and reactive maintenance operations. Meteorological forecasts on many time-scales are used to inform scheduling decisions, but are imperfect. Short-term maintenance scheduling is therefore a problem of decision-making under uncertainty. This paper proposes a probabilistic approach to the short-term scheduling problem based on a cost-loss model for individual maintenance missions, and probabilistic forecasts of appropriate access windows. This approach is found to increase the utilisation of possible access windows compared to using deterministic decision rules. The impact of forecasting on the availability and operational costs of offshore wind is then examined using a Monte Carlo-based cost model. This has quantified the impact on availability and revenue performance under a range of site conditions.
\end{abstract}

\section{INTRODUCTION}

Offshore wind farms provide renewable energy at a low marginal cost by harnessing the free and abundant wind resource found at sea. However, the high capital cost of turbines plus installation and maintenance in remote and hostile environments means that, with present technology, offshore wind energy is relatively expensive. Maintenance cost can represent $10 \%-30 \%$ of the cost of offshore wind energy, depending on technology type and distance from shore (Schwabe et al. 2011), due to the cost of chartering specialist vessels and unavailability caused by weather restrictions on safe maintenance access (Tavner 2012, Dinwoodie et al. 2013).

Maintenance logistics and strategies have received attention in the academic literature and motivated the development of many tools for analysing the impact of different decision-making practices on lifetime operational costs and the levelised cost of energy (Shafiee 2015). However, much of this work has focused on strategic decisions such as where to locate onshore bases, what number of maintenance vessels should be procured for a given wind farm, and so on. Much less work has focused on operational decision-making, such how to schedule work for the day-ahead given a weather forecast, and the impact of weather forecast errors on maintenance access.
Offshore maintenance operations are constrained by sea and wind conditions at every stage of a mission. Transit to and from site, personnel transfer to and from offshore structures, and the use of cranes, for example, are all subject to different limits for safe working (Tavner 2012). Operational models typically consider the impact of wind speed and significant wave height on accessibility; however, multi-wave criteria have been considered in (Sperstad, Halvorsen-Weare, Hofmann, Nonås, Stålhane, and Wu 2014). Maintenance operations may take a number of hours in addition to transit time from port. For this reason, weather forecast are essential when planning maintenance missions; however, this can only be done in the short-term since the skill of meteorological forecasts decreases with forecast horizon (Catterson et al. 2016, Buizza and Leutbecher 2015).

Planning and optimising seasonal campaigns and condition-based maintenance has been well studied; however, it is often assumed that all possible access windows are utilised (Shafiee 2015), which would require perfect weather forecasts to be realised in reality. The short-term decision-making problem of whether to attempt the next day's scheduled mission has received relatively little attention. Here we examine the decision that must be made a day-ahead and on the morning of a planned maintenance mission to decide whether to dispatch 
a vessel and attempt a maintenance operation or not.

Furthermore, since the value in restoring an unavailable turbine to operation is typically large relative to the cost of attempting a maintenance mission, there is a strong incentive for operators to schedule missions when the weather forecast is marginal, in the hope that actual weather conditions are better than forecast. Since the cost of success and failure are asymmetric, this decisionmaking problem requires a probabilistic treatment.

An optimal solution to this problem based on stochastic optimisation is proposed in (Besnard et al. 2011), and indicates that significant savings can be made compared to a deterministic; however, it is assumed that all scheduled tasks are successfully completed. Many others have examined the scheduling problem considering the uncertainty of asset health but assume perfect foresight of weather windows for maintenance access (Shafiee 2015).

Here, we consider short-term vessel scheduling decisions informed by day-ahead and longer term meteorological forecasts. We propose a simple costloss decision rule: first we predict the probability of success for the proposed mission, then compare that to the ratio of the cost of attempting the mission and the loss if the mission is abandoned. Probability of success is predicted using a logistic model with input features derived from day-ahead weather forecasts, and the cost-loss ratio is calculated based on expected lost energy capture until the next maintenance mission is successful based on five-day-ahead weather forecasts.

In Section 2 we describe the decision-making problem followed by the deterministic and probabilistic forecasting methodologies in Section 3. The wind farm cost model presented in Section 4. Results from a case studies based on six proposed UK offshore wind farms, broken down into forecast validation, analysis of the decision model and results from the Operational Expenditure (OPEX) model, are presented in Section 5. Finally, conclusions are drawn in Section 6.

\section{SHORT-TERM VESSEL SCHEDULING}

The day before a planned maintenance operation, a weather forecast is consulted to determine whether to attempt the planned mission or not. If the decision to proceed is taken, a crew will be assembled and vessel prepared the following morning. At this point a new weather forecast will be available containing more up-to-date information and the decision to attempt the mission may be revised. At each stage, the cost of attempting the mission and safety of the crew doing so must be considered and balanced against the potential loss of leaving a turbine unavailable or at risk of failure.



Figure 1: Flow chart for short-term vessel scheduling decisions.

This process is illustrated in Figure 1.

Regardless of the outcome of the decision making process, an access window either exists or does not. If the mission is cancelled and an access windows does exist, an opportunity to perform maintenance is been missed; likewise if the mission is attempted and an access window does not exist then costs associated with crew and fuel are incurred with no benefit.

When a maintenance mission is planned, crew are required prepare equipment and perform a number of safety checks during the early hours of the work day. There is therefore an incentive for operators to declare a weather day at the dayahead stage in order to utilise crew for onshore tasks during normal working hours and to maintain good morale.

If the forecast is close to safe operational limits the day is called marginal. Due to the low economic cost of preparing a vessel compared to the loss if the opportunity to restore a turbine to operation is missed, the decision to cancel a mission is typically left as late as possible, when more accurate forecasts are available.

\subsection{Deterministic Approach}

In the simplest case, the decision maker examines the relevant weather forecast and schedules a vessel if the forecast indicates that wind and wave conditions will be within safety limits for a sufficient period of time to perform the planned maintenance mission, and cancels the mission otherwise. On marginal days this approach is unsatisfactory 
since the risks and costs associated with attempting a mission are not part of the decision-making process.

\subsection{Probabilistic Approach}

The relative economic impact of the success and failure of a mission can be captured in the decisionmaking process by a cost-loss model. If $C$ is the cost of attempting a mission and $L$ is the loss if the mission is unsuccessful, then it is economic to attempt the mission if the probability of success $p$ is greater than $C / L$ (Thompson and Brier 1955).

The cost $C$ of attempting a mission is the combined fuel and personnel cost for the day. If a mission is attempted the vessel will travel from port to site and may wait for a number of hours until it is save to transfer technicians and equipment to the structure. For simplicity, we assume a value of $C=£ 20000$ for this study, following discussion with an offshore wind farm operator.

The loss $L$ is sum of 1 ) value of lost energy capture between this attempt and time at which the turbine successfully restored to operation, 2) the impact of a vessel being unavailable restore a different turbine in the future while it restores the first turbine, 3) the cost of attempting a second mission.

Lost energy capture is estimated using the fiveday-ahead forecast of wind and wave conditions. The down-time until the turbine is restored to operation and energy capture during that period are estimated deterministically. The value of the lost energy is then calculated using the wind turbine power curve and per-unit value of energy.

To implement this approach the probability of the a mission will be completed successfully must be forecast, and that is the subject of the next section.

\section{ACCESS FORECASTING}

Meteorological forecasts of many variables are available to wind farm operators and are used to inform scheduling decisions; however, interpreting and deriving value from the forecast for specific decisions is the responsibility of the operator. Here, we introduce a deterministic approach, which simply compares the weather forecast to vessel capability in order to predict access windows, and a probabilistic approach which uses the information derived from weather forecast to predict the probability that and access window will exist.

\subsection{Deterministic Forecast}

Weather forecasts issued at the day-ahead and work-day decision stages indicate the expected wind speed and significant wave height for each hour of the shift. If a sufficient period of time does not exist during which both wind and wave conditions are forecast to be within safe operating conditions, the deterministic forecasts is for a weather day, and vice versa.

\subsection{Probabilistic Forecast}

To make a scheduling decision using the cost-loss model it is necessary to estimate the probability that an access window will exist, given the meteorological forecasts and safety constraints. Here, we use logistic regression, which is a class of generalised linear model (P. McCullagh 1989), but note that other approaches are possible.

Logistic regression allows the probability $p$ that an access window will exist to be estimated, conditional on some vector of explanatory variables $\mathbf{x}$. Formally,

$$
p=\operatorname{Pr}(W=1 \mid \mathbf{x})=\frac{1}{1+\exp (-\boldsymbol{\beta} \cdot \mathbf{x})}
$$

where $W=1$ denotes a shift containing an access window sufficient to complete the proposed mission, and $W=0$ the converse. The vector $\boldsymbol{\beta}$, which is the same size as $\mathbf{x}$, contains the model parameters to be estimated.

The explanatory variables contained in $\mathbf{x}$ are selected from a list of features derived from the meteorological forecast for a given shift. The list of features are mean and maximum wind speed, mean and maximum significant wave height, total number of hours within safety limits, and maximum number of consecutive hours within safety limits. The features chosen to be included in $\mathbf{x}$ are those which have statistical significance in an test model containing all features.

This process is automated to allow rapid model fitting for missions with specific wind, wave and time constraints. The model parameters $\boldsymbol{\beta}$ are determined by maximum likelihood estimation.

\section{OPEX MODEL}

As wind farms have increased in size and distance from shore, operating them in a similar manner to onshore farms is becoming increasingly impractical. In order to better understand the additional complexities and investigate novel operational strategies, a significant body of research into the area of offshore wind OPEX modelling has developed in the last 5-10 years and is summarised in (Hofmann 2011). Due to the large number of models, several modelling methodologies with varying degrees of fidelity have been applied to offshore wind O\&M problem. This investigation considers the influence of time-dependent decisions on the operational performance of wind 
farms. Therefore, a time-domain model that has sufficient complexity to capture day ahead and day of decisions in order to influence operations was necessary.

The model used here is fully described in (Dinwoodie 2014), and utilised in (Dinwoodie, McMillan, Revie, Lazakis, and Dalgic 2013). There is an associated uncertainty when representing any complex engineering process with a simulation model; however, the developed OPEX model has been extensively evaluated against an observed wind farm performance as well as through model to model verification in (Dinwoodie, Endrerud, Hofmann, Martin, and Sperstad 2015), which has established the reliability and capability of the model. In addition to the functionality described in (Dinwoodie 2014), the decision process illustrated in Figure 1 is added and considered for each working shift. The model simulates a shift based operation process using the observed site wind and wave characteristics to determine accessibility and power production values. Failures are based on a constant failure rate for this study and simulated via a timeseries Markov Chain Monte Carlo failure process with the repair process determined on available resources, accessibility for the shift and forecasting decision.

\section{CASE STUDY}

In this section the decision-making problem described above is modelled and analysed. Six proposed or existing UK offshore wind farms locations are considered, corresponding to available weather data for this study. The wind farm location, distance to operating port and wind turbine configurations are listed in Table 1 . In all cases, the 5MW wind turbine power curve specified in (Jonkman, Butterfield, Musial, and Scott 2009) was scaled to calculate power production with a baseline value of $105 £ / M W h$ based on the upper value for offshore wind in the recent UK contract for difference auction. Only unscheduled corrective maintenance has been considered with 1 vessel per 30 turbines available for maintenance, regardless of distance to shore or turbine rating.

There is a complex relationship between wind farm configuration and operating environment and the resulting O\&M requirement for offshore wind. For for this study, only expected wind farm configuration and wind and wave characteristics have been changed between different site simulations. Other variables such as capability and types vessels, maintenance strategy, variation in reliability and issues around major repairs were kept constant and are based on current operational practice at UK wind farms. Therefore, any changes in forecasting performance and influence in availability are influenced only by the wind farm location
Table 1: List of proposed wind wind farms used in the case study. Location specifies the grid point for which weather data has been extracted, distance to shore, size and type of turbine are based on current design or application.

\begin{tabular}{lccc}
\hline Site & Location & $\begin{array}{c}\text { Distance } \\
(\mathrm{km})\end{array}$ & Turbines \\
\hline $\begin{array}{l}\text { Firth of } \\
\text { Forth }\end{array}$ & $56.27^{\circ} \mathrm{N} 1.87^{\circ} \mathrm{W}$ & 41.2 & $75 \times 7 \mathrm{MW}$ \\
Dogger Bank & $55.08^{\circ} \mathrm{N} 1.74^{\circ} \mathrm{E}$ & 165.6 & $200 \times 8 \mathrm{MW}$ \\
East Anglia & $52.57^{\circ} \mathrm{N} 2.62^{\circ} \mathrm{E}$ & 56.7 & $102 \times 8 \mathrm{MW}$ \\
Moray Firth & $58.19^{\circ} \mathrm{N} 2.74^{\circ} \mathrm{W}$ & 93.3 & $93 \times 7 \mathrm{MW}$ \\
Beatrice & $58.11^{\circ} \mathrm{N} 3.05^{\circ} \mathrm{W}$ & 24.7 & $84 \times 7 \mathrm{MW}$ \\
Greater & $51.87^{\circ} \mathrm{N} 1.96^{\circ} \mathrm{E}$ & 66.5 & $140 \times 3.6 \mathrm{MW}$ \\
\hline
\end{tabular}

and turbine configuration. Further sensitivity analysis of additional operational parameters has been identified for future consideration.

This section is divided into four parts: 5.1) description of the meteorological data used in the case study, 5.2) verification of access window forecasting methodologies, 5.3) analysis of the results of the deterministic and probabilistic decisionmaking processes, 5.4) evaluation of the impact of the different approaches on wind farm OPEX.

\subsection{Description of Meteorological Forecasts}

Meteorological forecasts produced by the UK Met Office using the WAVEWATCH III model covering the three year period from 2013 to the end of 2015 have been used for this case study. Variables used are forecasts of wind speed and significant wave height issued 4 times a day at hourly resolution from 0-36 hours ahead, and 3 hour resolution from 36-120 hours ahead, as well as hourly resolution hindcasts from the same model which are used in the absence of measurements.

\subsection{Access Window Forecast Verification}

The probabilistic predictions of categorical events, such as whether an access window exists or not, can be evaluated using the Brier score, which is given by

$$
\text { Brier Score }=\frac{1}{N} \sum_{i=1}^{N}\left(p_{i}-W_{i}\right)^{2},
$$

where $N$ is the number of forecasts being evaluated. The Brier score rewards both reliability and confidence. The best score achievable is 0 if the correct outcome is forecast with complete confidence. Confident forecasts, i.e. those close to 0 or 1 , are rewarded with a lower Brier score than cautions perditions, i.e. close to 0.5 , if they are correct, and more heavily penalised if they are the 'wrong side' of 0.5 . 
The Brier score can be decomposed into reliability, resolution and uncertainty. Reliability is a measure of how close the forecast probabilities are to the proportion of positive outcomes, resolution is a measure of how much the forecast probabilities vary from the climatic average, and uncertainty measures the inherent uncertainty of the event being forecast. Mathematically these are given by

$$
\begin{aligned}
\text { Reliability } & =\frac{1}{N} \sum_{k=1}^{K} n_{k}\left(p_{k}-\bar{W}_{k}\right)^{2}, \\
\text { Resolution } & =\frac{1}{N} \sum_{k=1}^{K} n_{k}\left(\bar{W}_{k}-\bar{W}\right)^{2}, \\
\text { Uncertainty } & =\bar{W}(1-\bar{W}),
\end{aligned}
$$

where $N$ is the total number of forecasts issued, $K$ is the number of unique forecasts issued, and $n_{k}$ is the total number of times the $k^{\text {th }}$ unique forecast has been issued. The terms $\bar{W}$ and $\bar{W}_{k}$ are the mean outcome and the mean outcome conditional on the $k^{\text {th }}$ unique forecast being issued, respectively. Here, forecasts are binned into 20 'unique' forecast bins centred on values from 0 to 1 in increments of 0.05 .

The Brier scores and decompositions from the case study are tabulated in Table 2. The logistic regression beats the deterministic approach by all measures. The skill of the probabilistic forecast is further evaluated using a reliability diagram in Figure 2. The logistic model is illustrated by the diagonal line and $95 \%$ confidence intervals, and the observed frequency of work days for each forecast probability bin is indicated by circular points. Underlaid bars illustrate the number of forecasts and observations contained in each bin on a logarithmic scale.

The majority of forecasts are in the $p<0.025$ and $p \geq 0.975$ bins which contain $15 \%$ and $65 \%$ for forecasts, respectively, with $6.6 \%$ of forecasts between $p=0.25$ and $p=0.75$.

This probabilistic prediction allows the forecast user to discriminate between marginal forecasts analytically and determine, based on the value of individual maintenance operation, whether to go ahead with the planned mission or not.

\subsection{Evaluation of Cost-Loss Model}

The number of work days realised, false positive (mission is attempted but fails due to weather) and false negative (mission is cancelled but would not have been prevented by adverse weather) work day forecasts for the deterministic and the probabilistic (logistic/cost-loss) decision schemes are presented in Table 3. In addition, results for 'perfect' forecasting are also presented to serve as a bench mark.

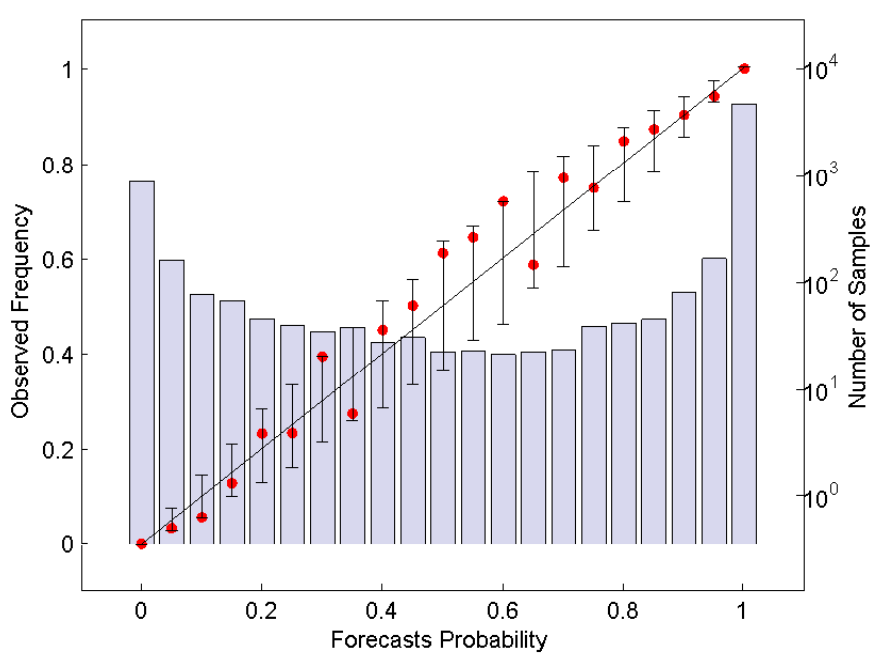

Figure 2: Reliability diagram for probabilistic forecast of work days with a wind limit of $12 \mathrm{~ms}^{-1}$, significant wave height limit of $1.5 \mathrm{~m}$, and minimum window length of 3 hours. Points indicate the frequency of work days for each forecast probability with $95 \%$ confidence intervals for the logistic model. The number of forecasts in each bin is indicated by the bar chart and the logarithmic scale on the right-hand vertical axis.

In this case, the decision scheme is the same as for deterministic forecasting, but with perfect foresight of access windows.

The deterministic forecast results in an average of $96 \%$ of possible access windows being utilised. A significant portion of access windows are therefore not utilised. For all locations the probabilistic approach results in more work days being realised than the deterministic method. In addition, the probabilistic approach results in a greater number of false positives reflecting occasions when missions with a low chance of success are attempted because the cost-loss ratio indicates that this is the correct economic decision. The full economic impact of the different decision-making procedures is evaluated in the next section.

The sensitivity of the number of days worked to the value of energy has been investigated. The perfect and deterministic decision making schemes are not influenced by the value of energy, whereas decision making based on the cost-loss model is since the lost revenue from an unavailable turbine is proportional to the value of energy. The results are illustrated in Figure 3 and show that as the value of energy increases, the increased incentive to restore an unavailable turbine to operation is sufficient to attempt riskier missions, some of which are successful. The value of energy in Figure 3 ranges from the $80-150 £ / M W h$ covering the full range of existing and expected future subsidy agreements in the UK at the time of writing. The impact of this sensitivity on operational performance is considered in Section5.4. 
Table 2: Briar score and its decomposition for deterministic and probabilistic forecasts of work days with a wind limit of $12 \mathrm{~ms}^{-1}$, significant wave height limit of $1.5 \mathrm{~m}$, and minimum window length of 3 hours.

\begin{tabular}{lrrrr}
\hline Method & Briar Score & Reliability & Resolution & Uncertainty \\
\hline Deterministic & 0.0427 & 0.0031 & 0.1498 & 0.1894 \\
Logistic Regression & 0.0296 & 0.0005 & 0.1602 & 0.1894 \\
\hline
\end{tabular}

Table 3: Results of decision model with perfect $(\mathrm{P})$, deterministic (D), and logistic (L) forecasts with a value of energy of $105 £ /$ MWh. The total number of events in the three-year test period are tabulated. Days Utilised gives the number of days successfully worked; False Positives are the number of missions that were attempted but failed because of adverse weather; False Negatives are missed opportunities.

\begin{tabular}{lrrrrrrrrr}
\hline & \multicolumn{3}{c}{ Days Utilised } & \multicolumn{3}{c}{ False Positives } & \multicolumn{3}{c}{ False Negatives } \\
Site & P & D & L & P & D & L & P & D & L \\
\hline Firth of Forth & 800 & 760 & 781 & 0 & 7 & 16 & 0 & 40 & 19 \\
Dogger Bank & 649 & 612 & 618 & 0 & 11 & 17 & 0 & 37 & 31 \\
East Anglia & 843 & 816 & 821 & 0 & 3 & 12 & 0 & 27 & 22 \\
Moray Firth & 834 & 790 & 803 & 0 & 5 & 12 & 0 & 44 & 31 \\
Beatrice & 902 & 866 & 874 & 0 & 6 & 6 & 0 & 36 & 28 \\
Greater Gabbard & 935 & 903 & 908 & 0 & 5 & 10 & 0 & 32 & 27 \\
\hline
\end{tabular}

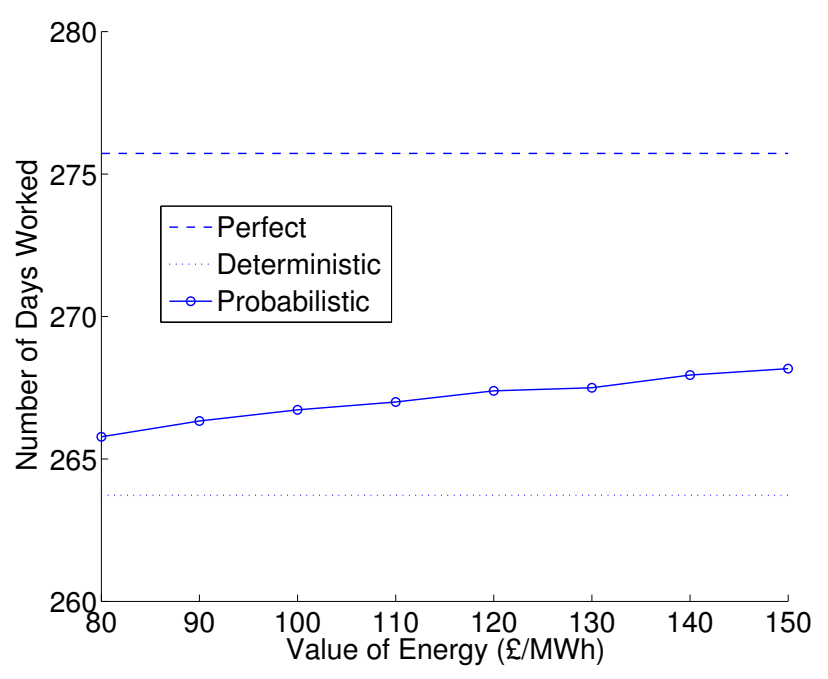

Figure 3: Mean number of days worked per year across all sites for each decision-making method plotted against value of energy. A high value of energy increases the value of missions and makes it economic to attempt missions with a lower probability of success. The result is an increase in both days worked and false positives.

\subsection{OPEX Model Results}

\subsubsection{Multi site analysis}

Monte Carlo simulation studies were carried out for each site and decision-making scheme in order to assess the impact of the different decision schemes on availability and revenue. The results of this are shown in Table 4 including the standard deviation across simulations.

A number of observations can be made from Table 4. Considering the deterministic forecast against the perfect forecasting case, failing to include the effects of forecasting in the OPEX model results in an over-estimation in operating performance in the range of $0.061-1.538 \%$ with an associated annual over-estimation of $220,000 £ / y r-$ $13.4 \mathrm{~m} £ / y r$. The extreme case of a very far from shore site such as Dogger Bank is unlikely to be operated from port and it is likely that this overestimates the impact from forecasting. Discount- ing this result, the average $\delta$ values between perfect and simple forecast for availability and annual revenue across the remaining sites are $0.21 \%$ and $830,000 £ / y r$, respectively. This is a significant result identifying that there is an important financial and operational cost associated with forecasting. Additionally, failing to consider forecasting within OPEX models will result in optimistic performance estimation.

Comparing the results from the logistics forecast and simple forecast in Table 4 , it is possible to quantify the benefit associated with improved forecasting techniques for each location. The improvements over the deterministic forecast range from $0.027-0.24 \%$ with a value of $112,000 £ / y r-$ $1.18 \mathrm{~m} £ / y r$. This makes it possible to perform a cost-benefit analysis on various forecasting approaches in order to determine if there is an economic case for implementing the techniques at that particular site. There is no direct correlation between the difference in perfect and simple forecast and the difference between logistic and simple forecast. The site where utilisation of a logistic forecast provides the greatest benefit is not the far offshore case. This identifies that the benefit from advanced forecasting approaches should be considered for all sites in order to determine the merit of the approach and not assumed to only provide value at large, far from shore sites.

The differences between perfect, deterministic forecast, and logistic methods varies between sites, the principal difference being distance to shore, as displayed in Table 3. Both wind/wave characteristics and the skill of the meteorological forecast are affected by the distance to shore. Further investigation to quantify the relative importance of these contributing factors has been identified for future analysis.

\subsubsection{Sensitivity to Value of Energy}

In order to investigate the impact that value of energy has on availability and revenue performance 
Table 4: Results of OPEX Simulation model with perfect (P), deterministic (D), and logistic (L) forecasts. Absolute values, deviation from the ideal case and standard deviation arising from the Monte Carlo simulation process are tabulated. Availability is the mean proportion of time that wind turbines are operational.

\begin{tabular}{lcccccccccc}
\hline & \multicolumn{2}{c}{ Availability $(\%)$} & \multicolumn{3}{c}{ Availability $\sigma$} & \multicolumn{2}{c}{$\Delta$ Availability } & \multicolumn{2}{c}{$\Delta$ Revenue/yr } \\
Site & $\mathrm{P}$ & $\mathrm{D}$ & $\mathrm{L}$ & $\mathrm{P}$ & $\mathrm{D}$ & $\mathrm{L}$ & $\mathrm{D}$ & $\mathrm{L}$ & $\mathrm{D}$ & $\mathrm{L}$ \\
\hline Beatrice & 95.83 & 95.58 & 95.62 & 0.098 & 0.094 & 0.104 & 0.250 & 0.217 & $£ 870 \mathrm{k}$ & $£ 650 \mathrm{k}$ \\
Dogger Bank & 70.04 & 68.50 & 68.64 & 0.346 & 0.357 & 0.328 & 1.538 & 1.405 & $£ 13.58 \mathrm{~m}$ & $£ 12.44 \mathrm{~m}$ \\
East Anglia & 94.46 & 94.18 & 94.42 & 0.155 & 0.150 & 0.150 & 0.275 & 0.035 & $£ 1.39 \mathrm{~m}$ & $£ 210 \mathrm{k}$ \\
Firth of Forth & 95.02 & 94.89 & 95.00 & 0.111 & 0.111 & 0.109 & 0.130 & 0.021 & $£ 420 \mathrm{k}$ & $£ 310 \mathrm{k}$ \\
Greater Gabbard & 96.28 & 96.22 & 96.25 & 0.051 & 0.054 & 0.052 & 0.061 & 0.035 & $£ 220 \mathrm{k}$ & $£ 80 \mathrm{k}$ \\
Moray Firth & 95.83 & 95.62 & 95.58 & 0.098 & 0.094 & 0.104 & 0.250 & 0.217 & $£ 870 \mathrm{k}$ & $£ 650 \mathrm{k}$ \\
\hline
\end{tabular}

Table 5: Sensitivity of OPEX model to logistic forecast cost function. Availability is the mean proportion of time that wind turbines are operational. Accessibility is the proportion of shift that can be worked, i.e. a mission is attempted and weather window exists.

\begin{tabular}{|c|c|c|c|}
\hline Site & $\begin{array}{l}\text { Value of } \\
\text { Energy }\end{array}$ & $\begin{array}{c}\text { Availability } \\
(\%)\end{array}$ & $\begin{array}{c}\text { Accessibil- } \\
\text { ity }(\%)\end{array}$ \\
\hline \multirow{3}{*}{$\begin{array}{l}\text { Moray } \\
\text { Firth }\end{array}$} & $85 £ / \mathrm{MWh}$ & 91.81 & 63.97 \\
\hline & $105 £ / M W h$ & 91.77 & 63.70 \\
\hline & $145 £ / M W h$ & 91.73 & 63.15 \\
\hline \multirow{3}{*}{$\begin{array}{l}\text { Dogger } \\
\text { Bank }\end{array}$} & $85 £ / M W h$ & 68.66 & 54.25 \\
\hline & $105 £ / M W h$ & 68.64 & 53.70 \\
\hline & $145 £ / M W h$ & 68.61 & 53.52 \\
\hline
\end{tabular}

when using the probabilistic decision scheme, an additional case study has been carried out. For this study, the value of energy was set to $85 £ / \mathrm{MWh}$, $105 £ / \mathrm{Mwh}$ and $145 £ / \mathrm{MWh}$ at the two sites where the logistic forecast was shown to have the greatest potential saving in the first case study. The results are shown in Table 5 .

It can be seen that the mean difference between the high and low cases for the two sites considered is $0.065 \%$. A direct economic analysis of this impact is not possible due to the different value of production for each of the cases. However, if the value of production is set to the baseline value of $105 £ /$ Mwh in post processing, this corresponds to an estimated revenue difference of 300$600,000 £ / y r$. Further analysis into the sensitivity of the logistic forecast under different operating conditions and comparison with alternative forecasting techniques will provide additional insights into the potential value from improved forecasting. In order to fully quantify this benefit, a cost associated with the implementation of the forecasting techniques should also be considered.

\section{CONCLUSIONS}

The use of access forecasts in short-term vessel scheduling decisions has been studied for the case of offshore wind. It is found that forecasting has a significant impact on the number of work days available to operators with an average of $4 \%$ of possible maintenance opportunities missed, a significant portion in the context of lifetime availability. Secondly, it is shown that a probabilistic approach to decision-making can increase the portion of days worked.
A method for forecasting the probability that a maintenance access window will exist has been presented. The approach, which uses meteorological forecasts as an input to a logistic regression model, allows the forecast user to discriminate between marginal forecasts and can serve as an input to a analytical decision-making tools.

A cost-loss decision model is proposed to inform scheduling decisions based on the probability of success given a weather forecast, and the value of maintenance operations in terms of forecast lost energy. This approach is shown to increase the proportion of access windows that are utilised for maintenance compared to a deterministic approach resulting in fewer missed opportunities at the cost of slightly increased number of missions which were attempted but failed due to poor weather.

The impact of different scheduling decision schemes has then been tested via an OPEX simulation in order to quantify the economic value of access forecasting and associate decision making. Discounting the very far from shore case, the performance impact associated with simple forecasting and decision rules was determined to be a $0.21 \%$ availability reduction and $830,000 £ / y r$ in lost revenue. In addition, probabilistic decision-making based a cost-loss model has been demonstrated to reduce this impact by up to $300,000 £ / y r$.

\section{ACKNOWLEDGEMENTS}

The authors thank the UK Met Office for the supply of meteorological data. Jethro Browell is supported by the University of Strathclyde's EPSRC Doctoral Prize, grant number EP/M508159/1. The baseline OPEX model was developed under the EPSRC Grant No.EP/G037728/1, Centre for Doctoral Training in Wind Energy Systems.

\section{REFERENCES}

Besnard, F., M. Patriksson, A. Stromberg, A. Wojciechowski, K. Fischer, and L. Bertling (2011, June). A stochastic model for opportunistic maintenance planning of offshore wind farms. In IEEE PowerTech, Trondheim, pp. 1-8. 
Buizza, R. and M. Leutbecher (2015). The forecast skill horizon. Quarterly Journal of the Royal Meteorological Society 141(693), 3366-3382.

Catterson, V. M., D. McMillan, I. Dinwoodie, M. Revie, J. Dowell, J. Quigley, and K. Wilson (2016). An economic impact metric for evaluating wave height forecasters for offshore wind maintenance access. Wind Energy 19(2), 199-212.

Dinwoodie, I. (2014). Modelling the operation and maintenance of offshore wind farms. Ph. D. thesis, University of Strathclyde, Glasgow.

Dinwoodie, I., O.-E. V. Endrerud, M. Hofmann, R. Martin, and I. B. Sperstad (2015). Reference cases for verification of operation and maintenance simulation models for offshore wind farms. Wind Engineering 39(1), 1-14.

Dinwoodie, I., D. McMillan, M. Revie, I. Lazakis, and Y. Dalgic (2013). Development of a combined operational and strategic decision support model for offshore wind. Energy Procedia 35, 157-166.

Hofmann, M. (2011). A review of decision support models for offshore wind farms with an emphasis on operation and maintenance strategies. Wind Engineering 35(1), 1-16.

Jonkman, J. M., S. Butterfield, W. Musial, and G. Scott (2009). Definition of a 5-mw reference wind turbine for offshore system development.

P. McCullagh, J. A. N. (1989). Generalized Linear Models (Second Edition ed.). Springer.

Schwabe, P., S. Lensink, and M. Hand (2011). Task 26: Multinational case study of financial cost of wind energy, work package 1, final report. Technical report, IEA Wind.

Shafiee, M. (2015). Maintenance logistics organization for offshore wind energy: Current progress and future perspectives. Renewable Energy 77, 182 - 193.

Sperstad, I. B., E. E. Halvorsen-Weare, M. Hofmann, L. M. Nonås, M. Stålhane, and M. Wu (2014). A comparison of single- and multi-parameter wave criteria for accessing wind turbines in strategic maintenance and logistics models for offshore wind farms. Energy Procedia 53, 221-230. \{EERA\} DeepWind' 2014, 11th Deep Sea Offshore Wind R\&amp;D Conference.

Tavner, P. (2012). Offshore Wind Turbines: reliability, availability and maintenance. IET Renewable Energy Series.

Thompson, J. C. and G. W. Brier (1955). The economic utility of weather forecasts. Monrthly Weather Review 83(11), 249-254. 